\title{
FOXA2 promotes the proliferation, migration and invasion, and epithelial mesenchymal transition in colon cancer
}

\author{
BAOLEI WANG ${ }^{1}$, GUANGWEI LIU $^{2}$, LEI DING ${ }^{3}$, JUN ZHAO $^{4}$ and YUN LU ${ }^{1}$ \\ ${ }^{1}$ Department of Gastrointestinal Surgery, The Affiliated Hospital of Qingdao University, Qingdao, \\ Shandong 266555; ${ }^{2}$ Emergency Department; ${ }^{3}$ Medical Administration Division; ${ }^{4}$ Department of Pharmacy, \\ The Affiliated Hospital of Qingdao University, Qingdao, Shandong 266003, P.R. China
}

Received September 10, 2017; Accepted April 24, 2018

DOI: $10.3892 / \mathrm{etm} .2018 .6157$

\begin{abstract}
The present study determined the expression and biological functions of FOXA2 gene in colon cancer in tissues, cells and animals. A total of 66 patients with colon cancer were included in the present study. Using The Human Protein Atlas database, expression and distribution of FOXA2 in colon cancer tissues were analyzed. Using immunohistochemistry, the expression and distribution of FOXA2 in colon cancer cells were studied. Reverse transcription-quantitative polymerase chain reaction (RT-qPCR) was performed to determine the expression of FOXA2 mRNA in colon cancer tissues. Following in vitro transfection with FOXA2 interference sequence (siR-FOXA2), the proliferation, cell cycle, migration and invasion of colon cancer HCT116 and HT29 cells were investigated using Cell Counting Kit-8 assay, flow cytometry, and Transwell assay, respectively. Furthermore, flow cytometry was used to determine apoptosis of HCT116 and HT29 cells. Western blotting was used to determine the expression of epithelial mesenchymal transition (EMT) proteins, E-Cadherin and Vimentin. Laser scanning confocal microscopy was performed to observe the cytoskeleton in HCT116 and HT29 cells. Results indicated tumorigenesis of colon cancer cells in nude mice. In addition, the expression of FOXA2 in colon cancer tissues was elevated and associated with the metastasis and clinical staging of colon cancer. Notably, inhibition of FOXA2 reduced the proliferation of colon cancer cells in vitro and reduced expression of FOXA2 was able to decrease the migration and invasion abilities of colon cancer cells. Furthermore, FOXA2 promoted EMT, inhibited apoptosis and enhanced the invasion ability of colon cancer cells. Decreased expression of FOXA2 inhibited tumorigenesis of colon cancer cells in nude mice. To conclude,
\end{abstract}

Correspondence to: Dr Yun Lu, Department of Gastrointestinal Surgery, The Affiliated Hospital of Qingdao University, 1677 Wutaishan Road, Qingdao, Shandong 266555, P.R. China E-mail: yanjiusheng2009@126.com

Key words: FOXA2, colon cancer, oncogene, proliferation, migration, invasion, epithelial mesenchymal transition the present study demonstrated that the expression of FOXA2 in colon cancer tissues was elevated and associated with the metastasis and clinical staging of colon cancer. As an oncogene, FOXA2 may promote the proliferation, migration and invasion and EMT in colon cancer.

\section{Introduction}

Colon cancer is a clinically common malignant tumor in digestive tract, and it has a very high incidence all over the world $(1,2)$. It is reported that the incidence of colon cancer is the third highest among all malignant tumors (3). At present, surgery combined with radiochemotherapy and immunotherapy are the main ways of clinical treatments for colon cancer (4-6). In patients with early-stage colon cancer, the degree of malignancy is not high, and lymph node metastasis does not occur (7). These patients usually have good postoperative recovery, and a 5-year survival rate of over $60 \%$ (8). By contrast, patients with advanced colon cancer usually have poor clinical treatment effects and a 5-year survival rate of $20 \%$, because of widespread metastasis and drug resistance (9). The invasion and metastasis of cancer is the main cause of death in colon cancer patients, but its molecular mechanism is still unclear. Forkhead box (Fox) family genes are abundant in fungi and animal cells, and their main function is to regulate gene expression by binding with DNA promoter region as a transcription factor (10). Fox family is first discovered to regulate embryonic development, and more and more studies show that Fox family proteins play a key regulatory role in immune system, cell cycle, energy metabolism, and cell aging $(11,12)$. FOXA2 gene, a member of Fox family, is localized at human chromosome 20p11, with a length of $45 \mathrm{~kb}(13,14)$. It contains 3 exons and 2 introns, and its protein product has 457 amino acids $(13,14)$. The amino acid sequence of its binding site at DNA is 5'-RYMAYAY-3' $(13,14)$. It is discovered that FOXA2 can bind to the promoter region of genes such as CREB and HNF6, thereby activating their transcription $(15,16)$. Under normal conditions, FOXA2 is expressed differently in liver, lungs, pancreas and other tissues, and its activity is regulated by phosphorylation and acetylation (17). It is also reported that the expression of FOXA2 is abnormal in many tumor tissues, and abnormal FOXA2 is involved in the processes of proliferation, invasion and metastasis, and epithelial mesenchymal 
transition (EMT) of various tumors, such as liver cancer, prostate cancer, stomach cancer, and bladder cancer $(18,19)$. At present, the expression and function of FOXA2 gene in colon cancer is still unclear.

EMT refers to a transformation process in which epithelial cells, under the influence by certain factors, lose the polarity of epithelial cells and acquire the characteristics of mesenchymal cells (20). This process is accompanied by changes in cell morphology, function, and gene expression profiles (21). EMT is a key step for tumor cells to break away from their original sites and gain the ability of invasion and metastasis, and alleviation of EMT suppresses invasion and metastasis of tumor cells (22). Therefore, it is necessary to study the molecular mechanism of tumor cell EMT. Studies show that a variety of transcription factors play important roles in tumor EMT, including ZEB1, E47 and Twist $(23,24)$. However, whether FOXA2 is involved in the regulation of EMT and thereby affecting tumor metastasis has not been reported before. In the present study, we investigate the expression and mechanism of action of FOXA2 in colon cancer at tissue and cellular levels.

\section{Materials and methods}

Patients. A total of 66 patients with colon cancer who received treatments at our hospital between January 2014 and December 2016 were included in the present study. Among the patients, 49 were males, and 17 were females, with an age range of 35-64 years and an average age of 48.5 years. The inclusion criterion was that the patients were initially diagnosed of colon cancer. The exclusion criteria were: i) the patient had other tumors; ii) the patient received radiotherapy or chemotherapy; iii) the patient had a long history of drug intake; iv) the patient had chronic diseases. None of the patients received radiochemotherapy before surgeries. The clinical and pathological data were collected, including tumor size, clinical stage, histological differentiation, lymph node metastasis and distant metastasis. Colon cancer tissues were collected from all patients. In addition, tumor-adjacent tissues were collected as control. All procedures were approved by the Ethics Committee of Qingdao University. Written informed consents were obtained from all patients or their families.

Cells. Normal colonic epithelial NCM460 cells, and colon cancer HCT116 and HT29 cells were cultured in DMEM medium supplemented with $10 \%$ fetal bovine serum, $100 \mathrm{IU} / \mathrm{ml}$ penicillin and $100 \mathrm{IU} / \mathrm{ml}$ streptomycin under $37^{\circ} \mathrm{C}, 5 \% \mathrm{CO}_{2}$, and $70 \%$ humidity. The cells were passaged every three days, and log-phase cells were collected for experiments.

One day before transfection, log-phase HCT116 and HT29 cells $\left(2 \times 10^{5}\right)$ were seeded onto 24 -well plates, and cultured in serum-free DMEM medium until reaching $70 \%$ confluency. In the first vial, $1.5 \mu 1$ FOXA2 siRNA [20 pmol/ $\mu 1$; FOXA2 interference sequence (siR-FOXA2) group] or $0.5 \mu \mathrm{g}$ FOXA2 plasmids (Hanbio Biotechnology Co., Ltd., Shanghai, China) was mixed with $50 \mu \mathrm{l}$ Opti Memi medium (Thermo Fisher Scientific, Inc., Waltham, MA, USA). In the second vial, $1 \mu 1$ Lipofectamine 2000 (Thermo Fisher Scientific, Inc.) was mixed with $50 \mu \mathrm{l}$ Opti-MEMI medium. After standing still for $5 \mathrm{~min}$, the two vials were combined for another waiting at room temperature for $20 \mathrm{~min}$. Then, the mixtures were added onto cells in respective groups. Six hours later, the medium was replaced with DMEM medium containing $10 \%$ fetal bovine serum. After cultivation at $37^{\circ} \mathrm{C}$ and $5 \% \mathrm{CO}_{2}$ for $48 \mathrm{~h}$, the cells were collected for further assays.

Immunohistochemical data analysis. After logging on The Human Protein Atlas database (http://www.proteinatlas.org/), the name of 'FOXA2' was searched to query the expression of FOXA2 in colon cancer and normal colon tissues. In human tissues, FOXA2 scores were given by the website. Search on the website showed that FOXA2 protein expression is up-regulated. Therefore, we tested protein and mRNA expression at cellular and tissue levels as shown below. In addition, positive rate and distribution were analyzed.

Reverse transcription-quantitative polymerase chain reaction (RT-qPCR). Colon cancer tissues and control tissues (100 mg) were ground into powder in liquid nitrogen and mixed with $1 \mathrm{ml}$ Trizol (Thermo Fisher Scientific, Inc.) for lysis. Then, total RNA was extracted using phenol chloroform method. The purity of RNA was determined by A260/A280 using ultraviolet spectrophotometry (Nanodrop ND2000; Thermo Fisher Scientific, Inc.). Then, cDNA was obtained by reverse transcription from $1 \mu \mathrm{g}$ RNA and stored at $-20^{\circ} \mathrm{C}$. Reverse transcription of was carried out using miScript II RT kit (Qiagen, Hilden, Germany).

For RT-qPCR, miScript SYBR ${ }^{\circledR}$ Green PCR Kit (Qiagen) was used. The RT-qPCR reaction system was composed of $10 \mu \mathrm{l}$ RT-qPCR-Mix, $0.5 \mu \mathrm{l}$ upstream primer (5'-CCCCTG AGTTGGCGGTGGT-3'), $0.5 \mu 1$ downstream primer (5'-TTG

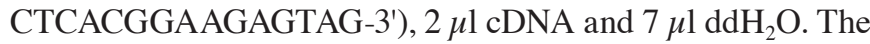
reaction protocol was: Initial denaturation at $95^{\circ} \mathrm{C}$ for $10 \mathrm{~min}$; 40 cycles of denaturation at $95^{\circ} \mathrm{C}$ for $1 \mathrm{~min}$ and annealing at $60^{\circ} \mathrm{C}$ for $30 \mathrm{sec}$

Western blotting. Cells $\left(1 \times 10^{6}\right)$ in each group were trypsinized and collected. Then, precooled radio immunoprecipitation assay (RIPA) lysis buffer $(1,000 \mu \mathrm{l} ; 50 \mathrm{mM}$ Tris-base, $1 \mathrm{mM}$ EDTA, $150 \mathrm{mM} \mathrm{NaCl,} \mathrm{0.1 \%} \mathrm{sodium} \mathrm{dodecyl} \mathrm{sulfate,}$ $1 \%$ Triton X-100, 1\% sodium deoxycholate; Beyotime Institute of Biotechnology, Shanghai, China) was added to the samples. After lysis for $30 \mathrm{~min}$ on ice, the mixture was centrifuged at $12,000 \mathrm{x} \mathrm{g}$ ta $4^{\circ} \mathrm{C}$ for $10 \mathrm{~min}$. The supernatant was used to determine protein concentration by bicinchoninic acid (BCA) protein concentration determination kit (RTP7102; Real-Times Biotechnology Co., Ltd., Beijing, China). Protein samples $(6 \mu \mathrm{g})$ were then mixed with $5 \mathrm{X}$ sodium dodecyl sulfate loading buffer before denaturation in boiling water bath for $10 \mathrm{~min}$. Afterwards, the samples $(10 \mu \mathrm{l})$ were subjected to $10 \%$ sodium dodecyl sulfate-polyacrylamide gel electrophoresis $(80 \mathrm{~V})$. The resolved proteins were transferred to polyvinylidene difluoride membranes on ice $(100 \mathrm{~V}, 1 \mathrm{~h})$ and blocked with $50 \mathrm{~g} / \mathrm{l}$ skimmed milk at room temperature for $1 \mathrm{~h}$. Then, the membranes were incubated with rabbit anti-human FOXA2 polyclonal primary antibody $(1: 1,000$; Abcam, Cambridge, UK), rabbit anti-human E-Cadherin polyclonal primary antibody (1:1,000; Abcam), rabbit anti-human Vimentin polyclonal primary antibody (1:1,000; Abcam), and rabbit anti-human GAPDH primary antibody (1:4,000; Abcam) at $4^{\circ} \mathrm{C}$ overnight. 
After extensive washing with phosphate-buffered saline with Tween-20 for 5 times of $5 \mathrm{~min}$, the membranes were incubated with goat anti-rabbit (1:2,000 for FOXA2, E-Cadherin and Vimentin) or goat anti-mouse (1:4,000 for GAPDH)-labelled horseradish peroxidase-conjugated secondary antibodies (Abcam) for $1 \mathrm{~h}$ at room temperature before washing with phosphate-buffered saline with Tween-20 for 5 times of $5 \mathrm{~min}$. Then, the membrane was developed with enhanced chemiluminescence detection kit (Sigma-Aldrich; Merck KGaA, Darmstadt, Germany) for imaging. Image lab v3.0 software (Bio-Rad Laboratories, Inc., Hercules, CA, USA) was used to acquire and analyze imaging signals. The relative contents of target proteins were expressed against GAPDH.

Cell Counting Kit-8 (CCK-8) assay. Cells were seeded at a density of 2,000/well in 96-well plates. At 0, 24, 48 and $72 \mathrm{~h}, 20 \mu \mathrm{l}$ CCK-8 (5 g/l) solution (Beyotime Institute of Biotechnology) was added onto the cells. On the last day, $150 \mu 1$ CCK-8 reaction solution was added and the cells were incubated at $37^{\circ} \mathrm{C}$ for $2 \mathrm{~h}$. The absorbance of each well was measured at $490 \mathrm{~nm}$ for plotting cell proliferation curves. Each group was tested in 3 replicate wells and the values were averaged.

Transwell assay. Matrigel was thawed at $4^{\circ} \mathrm{C}$ overnight and diluted with serum-free DMEM medium (dilution 1:3). The mixture $(50 \mu \mathrm{l})$ was evenly smeared into the upper chamber (HyClone; GE Healthcare Life Sciences, Logan, UT, USA) and incubated at $37^{\circ} \mathrm{C}$ for $1 \mathrm{~h}$. After solidification, $1 \times 10^{5}$ cells from each group were seeded into the upper chamber containing $200 \mu \mathrm{l}$ serum-free DMEM medium. In addition, $500 \mu 1$ DMEM medium supplemented with $10 \%$ fetal bovine serum was added into the lower chamber. After incubation at $37^{\circ} \mathrm{C}$ and $5 \% \mathrm{CO}_{2}$ for $24 \mathrm{~h}$, the chamber was removed and the cells in the upper chamber were wiped off. After being fixed with $4 \%$ formaldehyde for $10 \mathrm{~min}$, the membrane was stained using Giemsa method for microscopic observation of 5 random fields (magnification, $\mathrm{x} 200$ ). The number of transwell cells was calculated for the evaluation of cell invasion and migration ability. All procedures were carried out on ice with pipetting tips being precooled at $4^{\circ} \mathrm{C}$.

Flow cytometry. At $24 \mathrm{~h}$ after transfection, cells $\left(1 \times 10^{6}\right)$ in each group were washed with pre-cooled phosphate-buffered saline twice and subjected to cell cycle detection using Cycletest ${ }^{\mathrm{TM}}$ Plus DNA Reagent kit (BD Biosciences, Franklin Lakes, NJ, USA) following the manufacturer's manual. The data were analyzed using ModFit software (Verity Software House, Topsham, ME, USA).

Laser scanning confocal microscopy. At $24 \mathrm{~h}$ after transfection with siR-FOXA2, cells $\left(1 \times 10^{5}\right)$ in each group were seeded onto petri-dishes (diameter, $6 \mathrm{~cm}$ ) and incubated at $37^{\circ} \mathrm{C}$ and under $5 \% \mathrm{CO}_{2}$ for $24 \mathrm{~h}$. After discarding medium, the cells were washed with phosphate-buffered saline for three times, and fixed with $4 \%$ formaldehyde for $10 \mathrm{~min}$. After washing with phosphate-buffered saline for 3 times, the cells were stained with $5 \mu \mathrm{M}$ rhodamine for $5 \mathrm{~min}$. After additional washing with phosphate-buffered saline for 3 times, the cells were visualized under a laser scanning confocal microscope (SP8; Leica, Wetzlar, Germany).
In vivo assay in animal model. Sixteen BALB/C nude mice were divided evenly into two groups, negative control (NC) and siR-FOXA2 group. NC and siR-FOXA2 sequences were transfected into HCT116 cells using Lipofectamine 2000 (Thermo Fisher Scientific, Inc.) following the manufacturer's manual. At $24 \mathrm{~h}$ after transfection, the cells $\left(2 \times 10^{6}\right)$ in each group were trypsinized and resuspended with sterile phosphate-buffered saline $(0.2 \mathrm{ml})$. Then, the cell suspension was inoculated in nude mouse armpit to construct xenograft tumor model nude mice. Vital signs of nude mice were observed, and the mice were sacrificed in week 5 to extract tumor tissues. After fixation with $4 \%$ formaldehyde, the tumor tissues were dehydrated, paraffin-embedded, and sliced (4 nm thick) for immunohistochemistry test. E-Cadeherin and Vimentin rabbit anti-human polyclonal antibodies (Beyotime Institute of Biotechnology) were diluted at a ratio of 1:50 with water, and used to incubate the slices at $4^{\circ} \mathrm{C}$ overnight. After washing with phosphate-buffered saline twice, goat anti-rabbit secondary antibody was added, followed by incubation at $37^{\circ} \mathrm{C}$ for $30 \mathrm{~min}$ before color development. All animal experiments were conducted according to the ethical guidelines of Qingdao University (Qingdao, China).

Statistical analysis. Statistical analysis was performed using SPSS 17.0 software (IBM Corp., Armonk, NY, USA). The data were expressed as mean \pm standard deviation and intergroup comparison was carried out using Student's t-test. Comparison of multiple groups was performed using ANOVA followed by Tukey's or Dunnett's test. $\mathrm{P}<0.05$ was considered to indicate a statistically significant difference.

\section{Results}

Expression of FOXA2 in colon cancer tissues is elevated, and closely related with the metastasis and clinical staging of colon cancer. To examine the localization of FOXA2, we used immunohistochemistry. By comparing with public immunohistochemical database (http://www.proteinatlas.org/), we discovered that FOXA2 expression in colon cancer tissues was significantly higher than that in normal colon tissues (all data from Protein Atlas database; Fig. 1A). Immunohistochemical data showed that FOXA2 was localized in the nucleus of NCM460 cells, and the nucleus, cytoplasm and membrane of HCT116 and HT29 cells (Fig. 1B). To measure the expression of FOXA2, RT-qPCR was performed. The data showed that FOXA2 expression in colon cancer tissues was significantly higher than that in normal colon tissues (Fig. 1C). In addition, FOXA2 expression in colon cancer tissues from patients with lymphatic metastasis was significantly higher than that from patients without lymphatic metastasis (Fig. 1D). Clinical staging showed that FOXA2 level in colon cancer tissues at IV stage was significantly higher than those at I, II and III stages (Fig. 1E). These results suggest that the expression of FOXA2 in colon cancer tissues is elevated, and closely related with the metastasis and clinical staging of colon cancer.

Inhibition of FOXA2 reduces the proliferation of colon cancer cells in vitro. To determine the expression of FOXA2 protein, western blotting was used. The data showed that transfection 
A
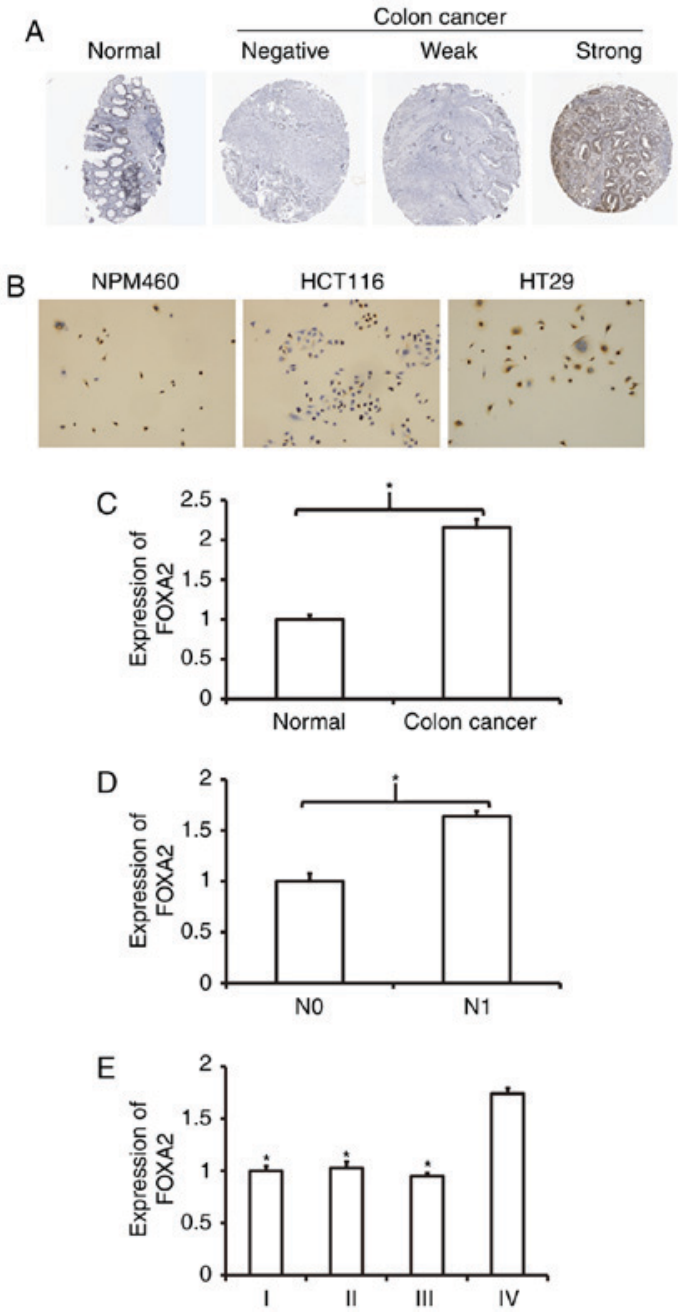

Figure 1.Expression and distribution of FOXA2 in colon cancer.(A) Expression of FOXA2 in colon cancer tissues shown by immunohistochemistry. The figure was obtained from the Human Protein Atlas database (https:/www.proteinatlas.org/ENSG00000125798-FOXA2/tissue/colon\#) (38). (B) Expression and distribution of FOXA2 in colon cancer HCT116 and HT29 cells. Magnification, x100. (C-E) Expression of FOXA2 mRNA in (C) normal and colon cancer tissues $\left({ }^{*} \mathrm{P}<0.05\right.$ compared with normal), (D) patients without/with lymphatic metastasis $\left({ }^{*} \mathrm{P}<0.05\right.$ compared with stage $\left.\mathrm{N} 0\right)$, or $(\mathrm{E})$ patients in different clinical stages (comparison of multiple groups was performed using ANOVA followed by Tukey's/Dunnett's test; ${ }^{*} \mathrm{P}<0.05$ compared with IV).

with siR-FOXA2 reduced the protein expression of FOXA2 in HCT116 and HT29 cells (Fig. 2A). To detect the proliferation of HCT116 and HT29 cells, CCK-8 assay was carried out. The data showed that the proliferation of HCT116 and HT29 cells transfected with siR-FOXA2 was significantly reduced compared with negative control group $(\mathrm{P}<0.05$; Fig. $2 \mathrm{~B})$. Flow cytometry showed that G1/S phase transition in HCT116 and HT29 cells transfected with siR-FOXA2 was reduced compared with negative control group (Fig. 2C). These results indicate that inhibition of FOXA2 reduces the proliferation of colon cancer cells in vitro, and suppresses the transition from G1 phase to $\mathrm{S}$ phase.

Reduced expression of FOXA2 is able to decrease the migration and invasion abilities of colon cancer cells. To determine the migration and invasion abilities of HCT116 and HT29 cells, Transwell assay was employed. The data showed that
A
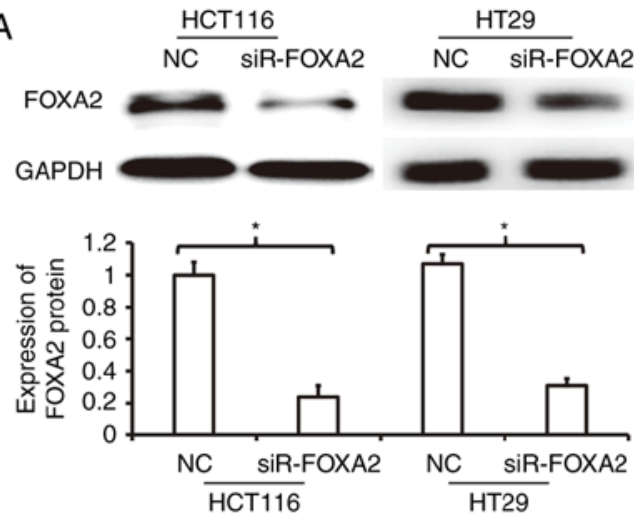

B
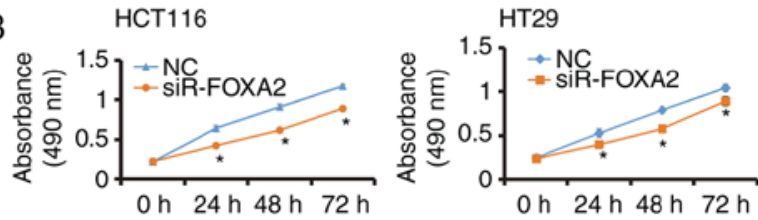

C
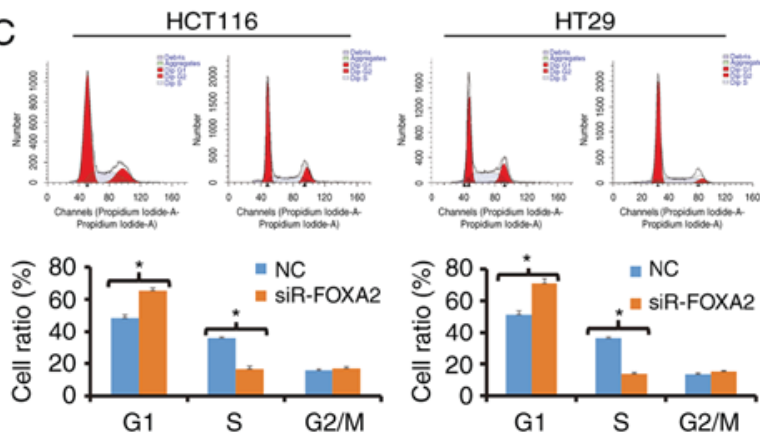

Figure 2. Effect of FOXA2 on the proliferation of colon cancer cells in vitro. (A) Expression of FOXA2 in HCT116 and HT29 cells transfected with FOXA2 interference sequence (siR-FOXA2). Western blotting was used to determine protein expression. ${ }^{*} \mathrm{P}<0.05$ compared with negative control (NC) as indicated. (B) Proliferation curves for HCT116 and HT29 cells transfected with siR-FOXA2. CCK-8 assay was performed to detect cell proliferation. ${ }^{*} \mathrm{P}<0.05$ compared with NC. (C) Percentages of cells in $\mathrm{G} 1, \mathrm{~S}$ or $\mathrm{G} 2 / \mathrm{M}$ phases. Flow cytometry was used to detect cell phases. ${ }^{*} \mathrm{P}<0.05$ compared with NC as indicated.

the number of transwell HCT116 cells after transfection with siR-FOXA2 was significantly reduced than that in negative control group in both migration and invasion assays $(\mathrm{P}<0.05$; Fig. 3). Similarly, the number of transwell HT29 cells after transfection with siR-FOXA2 was significantly lower than that in negative control group in both migration and invasion assays ( $\mathrm{P}<0.05$; Fig. 3). The results suggest that reduced expression of FOXA 2 is able to decrease the migration and invasion abilities of colon cancer cells.

FOXA2 may promote EMT, inhibit apoptosis, and enhance the invasion ability of colon cancer cells. To detect EMT, apoptosis and cytoskeleton, Western blotting, flow cytometry and laser scanning confocal microscopy were carried out, respectively. Western blots showed that the expression of E-Cadherin in HCT116 and HT29 cells transfected with siR-FOXA2 was significantly higher than that in NC group, while expression of Vimentin in HCT116 and HT29 cells transfected with siR-FOXA2 was lower than that in NC group (Fig. 4A). In the meantime, flow cytometry showed that the apoptosis of HCT116 

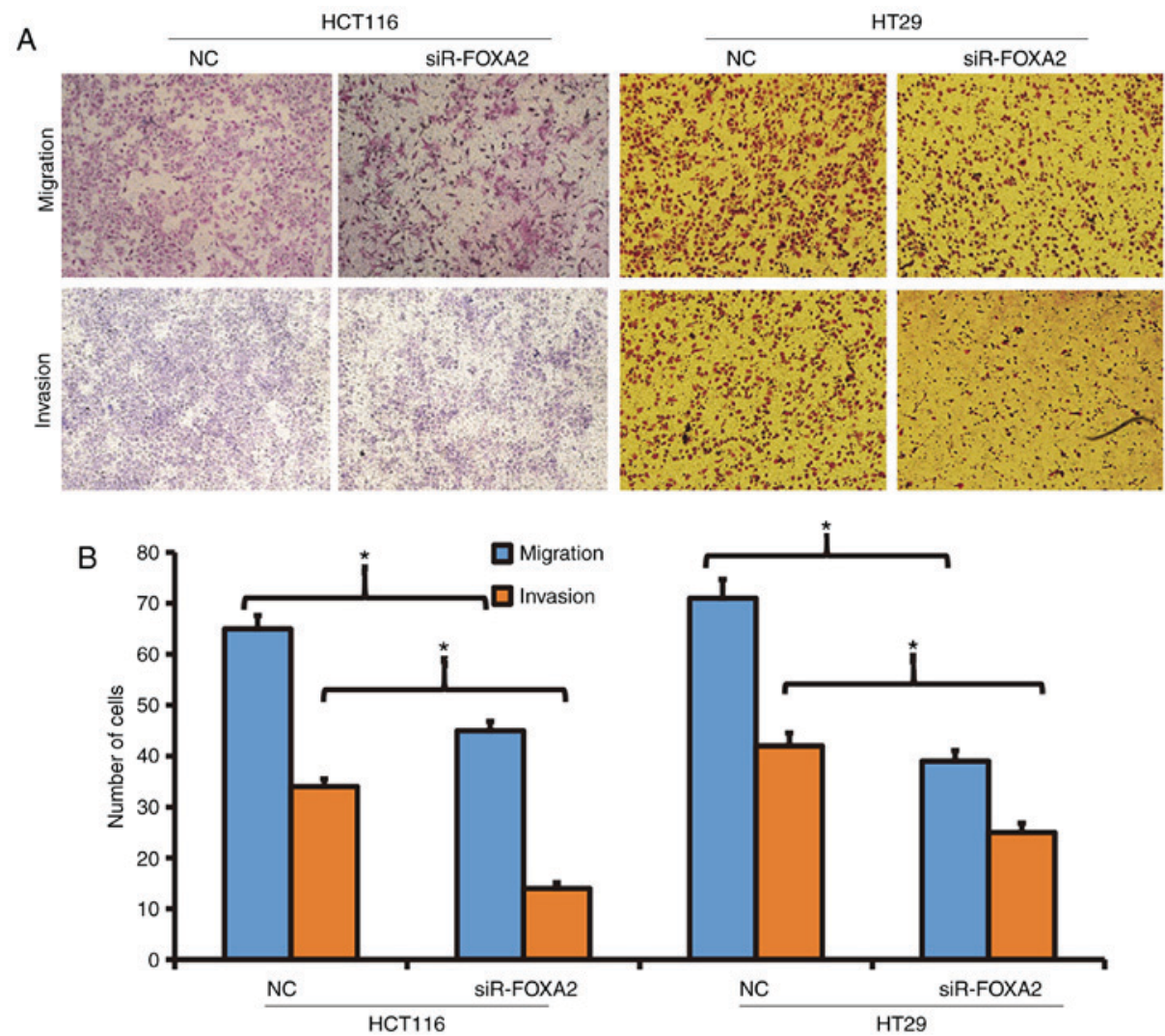

Figure 3. Effect of FOXA2 on the migration and invasion of colon cancer cells. (A) Images of HCT116 and HT29 cells transfected with FOXA2 interference sequence (siR-FOXA2). Magnification, x100. (B) Number of HCT116 and HT29 cells transfected with siR-FOXA2. Transwell assay was performed to determine migration and invasion abilities. ${ }^{*} \mathrm{P}<0.05$ compared with $\mathrm{NC}$ as indicated.
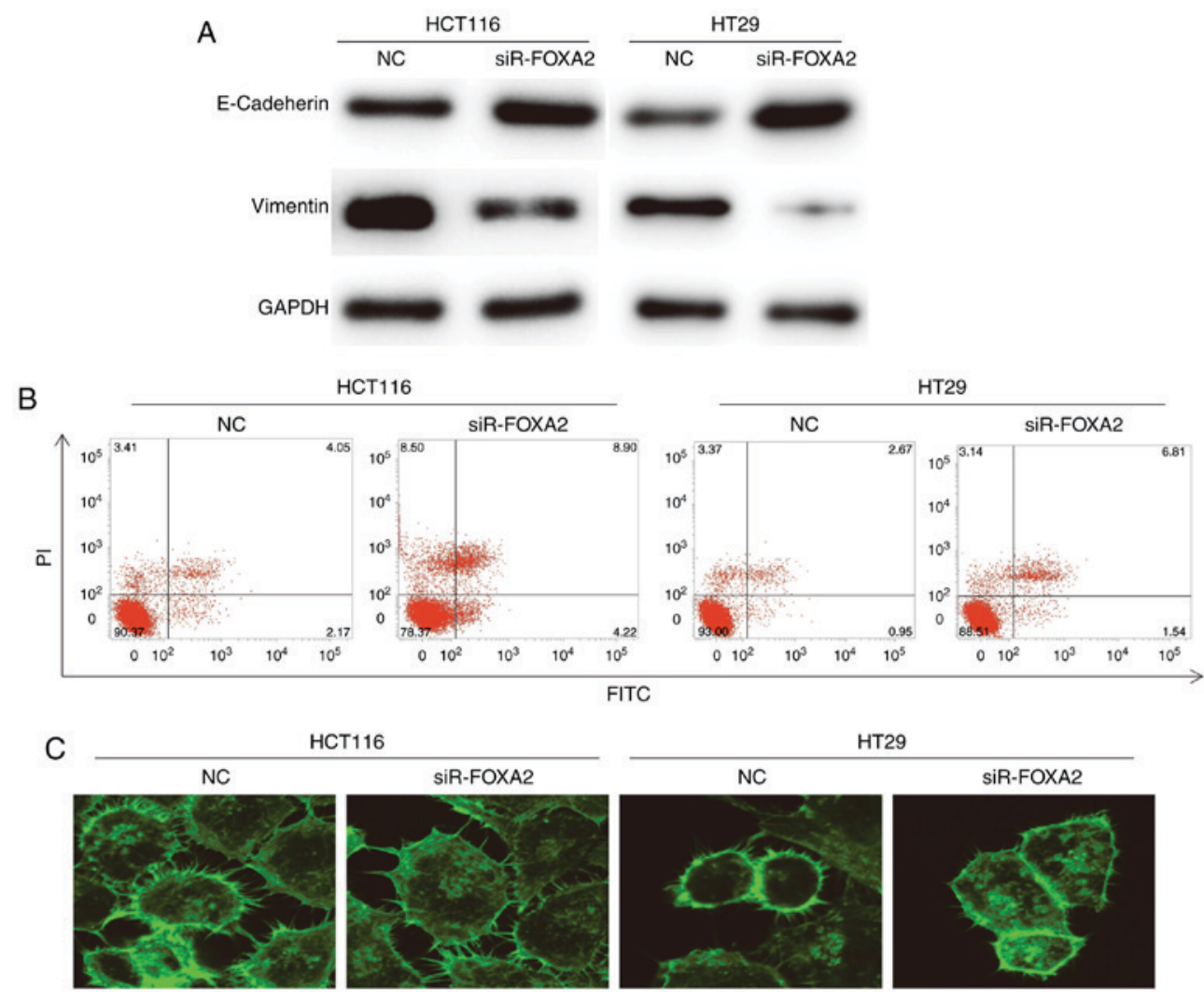

Figure 4. Effect of FOXA2 on epithelial mesenchymal transition of colon cancer cells. (A) Expression of E-Cadherin and Vimentin in HCT116 and HT29 cells transfected with FOXA2 interference sequence (siR-FOXA2). Western blotting was used to measure the expression of E-Cadherin and Vimentin. (B) Apoptosis of HCT116 and HT29 cells transfected with siR-FOXA2. Flow cytometry was performed to detect apoptosis. (C) Protrusion of the cell membrane in HCT116 and HT29 cells transfected with siR-FOXA2. Laser scanning confocal microscopy was used to observe the cells (magnification, x400). 
A
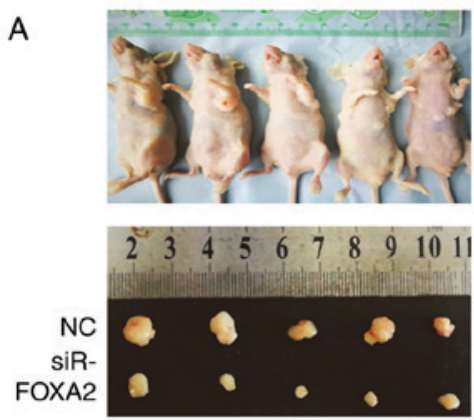

B

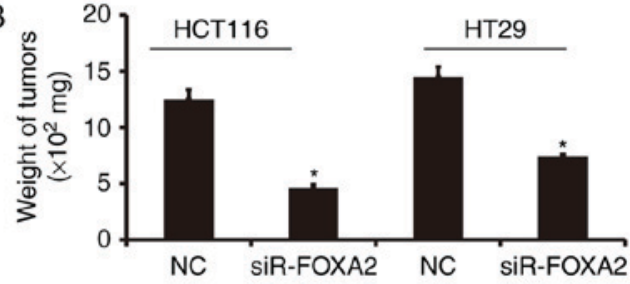

C

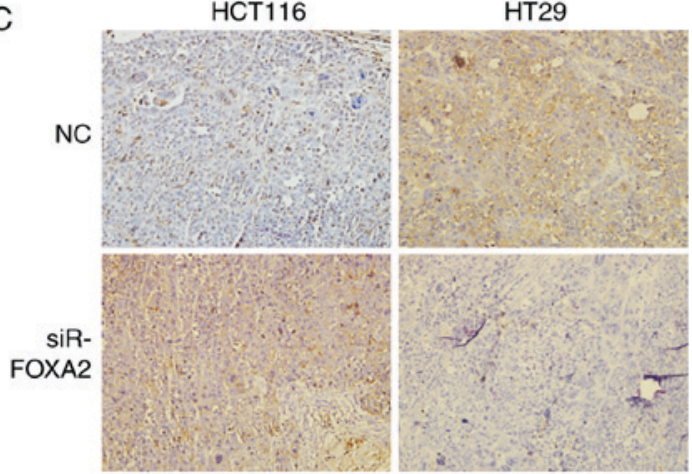

Figure 5. Effect of FOXA2 on tumorigenesis of colon cancer cells in nude mice. (A) Representative images of xenograft formed by HCT116 cells transfected with FOXA2 interference sequence (siR-FOXA2). (B) Weight of tumors from nude mice inoculated with HCT116 or HT29 cells that were transfected with siR-FOXA2. ${ }^{*}<0.05$ vs. negative control (NC). (C) Expression of E-Cadherin and Vimentin in xenograft tissues derived from HCT116 cells that were transfected with siR-FOXA2. Immunohistochemistry was used to detect the expression of E-Cadherin and Vimentin. Magnification, x200.

and HT29 cells transfected with siR-FOXA2 was enhanced than those of NC groups (Fig. 4B). Laser scanning confocal microscopy showed that protrusion of the cell membrane in HCT116 and HT29 cells transfected with siR-FOXA2 was reduced than that in NC group (Fig. 4C). These results indicate that FOXA2 may promote EMT, inhibit apoptosis, and enhance the invasion ability of colon cancer cells.

Decreased expression of FOXA2 inhibits tumorigenesis of colon cancer cells in nude mice. To test the effect of FOXA2 on the formation of colon tumor, nude mice were used. Tumor diameter in siR-FOXA2 group seemed smaller than that in NC group (Fig. 5A). Similarly, weights of colon tumors from mice inoculated with HCT116 or HT29 cells transfected with siR-FOXA2 were significantly lower than that in NC group, respectively ( $\mathrm{P}<0.05$; Fig. 5B). Immunohistochemistry showed that E-Cadherin expression in siR-FOXA2 group seemed higher than that in NC group, while Vimentin expression in in siR-FOXA2 group seemed lower than that in NC group (Fig. 5C). The results suggest that decreased expression of FOXA2 inhibits tumorigenesis of colon cancer cells in nude mice.

\section{Discussion}

Tumor recurrence and metastasis are the key factors that restrict the postoperative survival of colon cancer patients (25). Studies show that the recurrence and metastasis of colon cancer are closely related to gene expression, methylation, mutation, tumor stem cells, drug resistance and immunosuppression $(7,26)$. Transcription factors play key regulatory roles in the process of gene expression regulation, and they are important drug targets (27). In the present study, we discover that the expression of FOXA2 is up-regulated in colon cancer tissues, and correlated with lymphatic metastasis and clinical staging of colon cancer. In vitro and in vivo experiments show that FOXA 2 can promote the proliferation, migration and invasion of colon cancer cells, and EMT may be the key factor for FOXA2 to promote metastasis of colon cancer. These results suggest that FOXA2 plays an oncogene role by promoting EMT and colon cancer recurrence and metastasis.

Transcription factors can bind to the binding sites of the promoter region of genes, and initiate gene transcription, having important regulatory functions $(28,29)$. In cancer, transcription factors are often able to initiate transcription of multiple downstream oncogenes or tumor-suppressor genes, thereby inducing a cascade of responses that regulate cell proliferation, invasion, metastasis, differentiation, drug resistance, and apoptosis $(30,31)$. Studies show that multiple transcription factors are closely related to the recurrence and metastasis of colon cancer. For example, Ma et al discover that KLF4 inhibits the proliferation of colon cancer by activating NDRG2 expression (32). Stein et al discover that MACC1 indirectly activates the HGF/c-Met signaling pathway by activating transcription of c-Met gene, and promotes the recurrence and metastasis of colon cancer (33). In the present study, we find that transcription factor FOXA2 is up-regulated in colon cancer tissues. Moreover, our immunohistochemical data show that FOXA2 expression is distributed in the nucleus, cytoplasm and membrane in colon cancer, which is abnormal. Our RT-qPCR data demonstrate that expression of FOXA2 is up-regulated in colon cancer tissues, and positively correlated with lymphatic metastasis and clinical staging. These results indicate that FOXA2 is associated with the development and progression of colon cancer and may play the role of an oncogene.

Some studies show that the function of FOXA2 in tumors is two-sided. For example, Li et al report that miR-187 can target the expression of FOXA2 and promote the proliferation and metastasis of gastric cancer, suggesting that FOXA2 may be a tumor-suppressor gene in gastric cancer (34). Down-regulation of FOXA2 may promote EMT in pancreatic cancer, suggesting that FOXA2 may inhibit tumor EMT (35). Tu et al discover that miR-1291 inhibits proliferation and metastasis of pancreatic cancer by targeting FOXA2 (36). Our results in the present study show that FOXA2 expression is elevated in HCT116 and HT29 cells. CCK-8 assay shows that interference of FOXA2 expression reduces the proliferation of HCT116 and HT29 cells. In addition, HCT116 and HT29 cells in FOXA2 interference group have shown G1/S arrest, suggesting that down-regulation of FOXA2 inhibits the proliferation of colon cancer cells. Transwell assay shows that the number of cells that cross the membrane in FOXA2 interference group is lower than that in control group, suggesting that 
FOXA2 is able to promote the migration and invasion of colon cancer cells. Furthermore, our Western blotting data show that expression of E-Cadherin and Vimentin in FOXA2 interference group is up-regulated and down-regulated, respectively than that in control group, suggesting that EMT of colon cancer is inhibited by down-regulation of FOXA2. Of note, the apoptosis of cells in FOXA2 interference group is enhanced than control group, and laser scanning confocal microscopy shows that the cytoskeleton in the cell membrane of the FOXA2 interference group is more abundant, suggesting that these cells have stronger mobility. Tumor formation in nude mice shows that the growth rate of tumor in FOXA2 interference group is significantly lower than that in NC group. In addition, E-Cadherin expression is up-regulated and Vimentin expression is down-regulated in tumor tissues of FOXA2 interference group, suggesting that EMT is inhibited. These results indicate that FOXA2 promotes the proliferation, migration and EMT of colon cancer cells.

In conclusion, the present study demonstrates that FOXA2 is an oncogene in colon cancer, and the up-regulation of its expression promotes the proliferation, migration and invasion of colon cancer. As a transcription factor, up-regulation of FOXA2 expression has clinical values in the diagnosis and prognosis evaluation of colon cancer. Because FOXA2 initiates the expression of a large number of downstream genes, it can also be a potential diagnostic and therapeutic target for colon cancer, being consistent with a previous report (37). In future studies, we will investigate the molecular mechanisms of FOXA2 in colon cancer, such as downstream transcriptional genes of FOXA2 and relevant signaling pathways.

\section{Acknowledgements}

The authors wish to thank their department and research team for their help and dedication.

\section{Funding}

The present work was supported by the National Natural Science Foundation of China (grant no. 81172031).

\section{Availability of data and materials}

The analyzed data sets generated during the study are available from the corresponding author on reasonable request.

\section{Authors' contributions}

BW and YL designed the study; BW, GL, LD and JZ were involved in designing and performing experiments; BW, GL and YL analyzed the data. The final version of the manuscript has been read and approved by all authors, and each author believes that the manuscript represents honest work.

\section{Ethics approval and consent to participate}

All procedures performed in the current study were approved by the Ethics Committee of Qingdao University. Written informed consent was obtained from all patients or their families.

\section{Consent for publication}

Not applicable.

\section{Competing interests}

The authors declare that they have no competing interests.

\section{References}

1. Chen Y, Fang L, Li G, Zhang J, Li C, Ma M, Guan C, Bai F, Lyu J and Meng QH: Synergistic inhibition of colon cancer growth by the combination of methylglyoxal and silencing of glyoxalase I mediated by the STAT1 pathway. Oncotarget 8: 54838-54857, 2017.

2. Chino XMS, Martinez CJ, Garzón VRV, González IÁ, Treviño SV, Bujaidar EM, Ortiz GD and Hoyos RB: Cooked chickpea consumption inhibits colon carcinogenesis in mice induced with azoxymethane and dextran sulfate sodium. J Am Coll Nutr 36: 391-398, 2017

3. Cusimano A, Balasus D, Azzolina A, Augello G, Emma MR, Di Sano C, Gramignoli R, Strom SC, McCubrey JA, Montalto G and Cervello M: Oleocanthal exerts antitumor effects on human liver and colon cancer cells through ROS generation. Int $\mathrm{J}$ Oncol 51: 533-544, 2017.

4. Cho N, Ransom TT, Sigmund J, Tran T, Cichewicz RH, Goetz M and Beutler JA: Growth inhibition of colon cancer and melanoma cells by versiol derivatives from a paraconiothyrium species. J Nat Prod 80: 2037-2044, 2017.

5. Myint ZW and Goel G: Role of modern immunotherapy in gastrointestinal malignancies: A review of current clinical progress. J Hematol Oncol 10: 86, 2017.

6. Goel G and Sun W: Advances in the management of gastrointestinal cancers-an upcoming role of immune checkpoint blockade. J Hematol Oncol 8: 86, 2015.

7. Hou PC, Li YH, Lin SC, Lin SC, Lee JC, Lin BW, Liou JP, Chang JY, Kuo CC, Liu YM, et al: Hypoxia-induced downregulation of DUSP-2 phosphatase drives colon cancer stemness. Cancer Res 77: 4305-4316, 2017.

8. Pahwa M, Harris MA, MacLeod J, Tjepkema M, Peters PA and Demers PA: Sedentary work and the risks of colon and rectal cancer by anatomical sub-site in the Canadian census health and environment cohort (CanCHEC). Cancer Epidemiol 49: 144-151, 2017.

9. Múnera JO, Sundaram N, Rankin SA, Hill D, Watson C, Mahe M, Vallance JE, Shroyer NF, Sinagoga KL, Zarzoso-Lacoste A, et al: Differentiation of human pluripotent stem cells into colonic organoids via transient activation of BMP signaling. Cell Stem Cell 21: 51-64.e6, 2017.

10. Ancey PB, Ecsedi S, Lambert MP, Talukdar FR, Cros MP, Glaise D, Narvaez DM, Chauvet V, Herceg Z, Corlu A and Hernandez-Vargas H: TET-catalyzed 5-hydroxymethylation precedes HNF4A promoter choice during differentiation of bipotent liver progenitors. Stem Cell Reports 9: 264-278, 2017.

11. Von Stetina SE, Liang J, Marnellos G and Mango SE: Temporal regulation of epithelium formation mediated by FoxA, MKLP1, MgcRacGAP, and PAR-6. Mol Biol Cell 28: 2042-2065, 2017.

12. McFadden VC, Shalaby RE, Iram S, Oropeza CE, Landolfi JA, Lyubimov AV, Maienschein-Cline M, Green SJ, Kaestner KH and McLachlan A: Hepatic deficiency of the pioneer transcription factor FoxA restricts hepatitis B virus biosynthesis by the developmental regulation of viral DNA methylation. PLoS Pathog 13: e1006239, 2017.

13. Li J, Dantas Machado AC, Guo M, Sagendorf JM, Zhou Z, Jiang L, Chen X, Wu D, Qu L, Chen Z, et al: Structure of the forkhead domain of FOXA2 bound to a complete DNA consensus site. Biochemistry 56: 3745-3753, 2017.

14. Bastidas-Ponce A, Roscioni SS, Burtscher I, Bader E, Sterr M, Bakhti $\mathrm{M}$ and Lickert H: Foxa2 and Pdx1 cooperatively regulate postnatal maturation of pancreatic $\beta$-cells. Mol Metab 6: 524-534, 2017.

15. Rausa FM III, Hughes DE and Costa RH: Stability of the hepatocyte nuclear factor 6 transcription factor requires acetylation by the CREB-binding protein coactivator. J Biol Chem 279: 43070-43076, 2004. 
16. Zhang L, Rubins NE, Ahima RS, Greenbaum LE and Kaestner KH: Foxa2 integrates the transcriptional response of the hepatocyte to fasting. Cell Metab 2: 141-148, 2005.

17. LehnerF,KulikU,Klempnauer JandBorlakJ: Inhibition of the liver enriched protein FOXA2 recovers HNF6 activity in human colon carcinoma and liver hepatoma cells. PLoS One 5: e13344, 2010.

18. Berg DT, Gerlitz B, Sharma GR, Richardson MA, Stephens EJ, Grubbs RL, Holmes KC, Fynboe K, Montani D, Cramer MS, et al: FoxA2 involvement in suppression of protein $\mathrm{C}$, an outcome predictor in experimental sepsis. Clin Vaccine Immunol 13: 426-432, 2006.

19. Kanaki M, Tiniakou I, Thymiakou E and Kardassis D: Physical and functional interactions between nuclear receptor LXRo and the forkhead box transcription factor FOXA2 regulate the response of the human lipoprotein lipase gene to oxysterols in hepatic cells. Biochim Biophys Acta 1860: 848-860, 2017.

20. Li D, He C, Wang J, Wang Y, Bu J, Kong X and Sun D: MicroRNA-138 inhibits cell growth, invasion and EMT of non-small cell lung cancer via SOX4/p53 feedback loop. Oncol Res: Jun 13, 2017 (Epub ahead of print).

21. Zhou Q, Dai J, Chen T, Dada LA, Zhang X, Zhang W, DeCampMM, Winn RA, Sznajder JI and Zhou G: Downregulation of PKC $5 /$ Pard3/Pard6b is responsible for lung adenocarcinoma cell EMT and invasion. Cell Signal 38: 49-59, 2017.

22. Wahl GM and Spike BT: Cell state plasticity, stem cells, EMT, and the generation of intra-tumoral heterogeneity. NPJ Breast Cancer 3: 14, 2017.

23. Cao Z, Zheng X, Cao L and Liang N: MicroRNA-539 inhibits the epithelial-mesenchymal transition of esophageal cancer cells by twist-related protein 1-mediated modulation of melanoma associated antigen A4 (MAGEA4). Oncol Res: Jun 12, 2017 (Epub Ahead of Print).

24. Lazarova D and Bordonaro M: ZEB1 mediates drug resistance and EMT in p300-deficient CRC. J Cancer 8: 1453-1459, 2017.

25. Aquino RGF, Vasques PHD, Cavalcante DIM, Oliveira ALS, Oliveira BMK and Pinheiro LGP: Invasive ductal carcinoma: Relationship between pathological characteristics and the presence of axillary metastasis in 220 cases. Rev Col Bras Cir 44 163-170, 2017.

26. Laudato S, Patil N, Abba ML, Leupold JH, Benner A, Gaiser T, Marx A and Allgayer H: P53-induced miR-30e-5p inhibits colorectal cancer invasion and metastasis by targeting ITGA6 and ITGB1. Int J Cancer 141: 1879-1890, 2017.

27. Schayek H, Laitman Y, Katz LH, Pras E, Ries-Levavi L, Barak F and Friedman E: Colorectal and endometrial cancer risk and age at diagnosis in BLMAsh mutation carriers. Isr Med Assoc J 19: 365-367, 2017

28. Liu Z, Zhao Y, Fang J, Cui R, Xiao Y and Xu Q: SHP2 negatively regulates HLA-ABC and PD-L1 expression via STAT1 phosphorylation in prostate cancer cells. Oncotarget 8: 53518-53530, 2017.
29. Nelson AC, Cutty SJ, Gasiunas SN, Deplae I, Stemple DL and Wardle FC: In vivo regulation of the zebrafish endoderm progenitor niche by T-Box transcription factors. Cell Rep 19: 2782-2795, 2017.

30. Shen Y, Nar R, Fan AX, Aryan M, Hossain MA, Gurumurthy A, Wassel PC, Tang M, Lu J, Strouboulis J and Bungert J: Functional interrelationship between TFII-I and E2F transcription factors at specific cell cycle gene loci. J Cell Biochem 119: 712-722, 2018.

31. Seo HS, Ku JM, Choi HS, Woo JK, Lee BH, Kim DS, Song HJ, Jang BH, Shin YC and Ko SG: Apigenin overcomes drug resistance by blocking the signal transducer and activator of transcription 3 signaling in breast cancer cells. Oncol Rep 38: 715-724, 2017

32. Ma Y, Wu L, Liu X, Xu Y, Shi W, Liang Y, Yao L, Zheng J and Zhang J: KLF4 inhibits colorectal cancer cell proliferation dependent on NDRG2 signaling. Oncol Rep 38: 975-984, 2017.

33. Stein U, Walther W, Arlt F, Schwabe H, Smith J, Fichtner I, Birchmeier W and Schlag PM: MACC1, a newly identified key regulator of HGF-MET signaling, predicts colon cancer metastasis. Nat Med 15: 59-67, 2009.

34. Li C, Lu S and Shi Y: MicroRNA-187 promotes growth and metastasis of gastric cancer by inhibiting FOXA2. Oncol Rep 37: 1747-1755, 2017.

35. Kondratyeva LG, Sveshnikova AA, Grankina EV, Chernov IP, Kopantseva MR, Kopantzev EP and Sverdlov ED: Downregulation of expression of mater genes SOX9, FOXA2, and GATA4 in pancreatic cancer cells stimulated with TGF $\beta 1$ epithelial-mesenchymal transition. Dokl Biochem Biophys 469: 257-259, 2016.

36. Tu MJ, Pan YZ, Qiu JX, Kim EJ and Yu AM: MicroRNA-1291 targets the FOXA2-AGR2 pathway to suppress pancreatic cancer cell proliferation and tumorigenesis. Oncotarget 7: 45547-45561, 2016.

37. Jayachandran A, Dhungel B and Steel JC: Epithelial-to-mesenchymal plasticity of cancer stem cells: Therapeutic targets in hepatocellular carcinoma. J Hematol Oncol 9: 74, 2016

38. Uhlén M, Fagerberg L, Hallström BM, Lindskog C, Oksvold P, Mardinoglu A, Sivertsson A., Kampf C, Sjöstedt E, Asplund A, et al: Proteomics. Tissue-based map of the human proteome. Science 347: 1260419, 2015.

This work is licensed under a Creative Commons Attribution-NonCommercial-NoDerivatives 4.0 International (CC BY-NC-ND 4.0) License. 